\title{
Streams fish from Upper Araguaia and Middle Rio da Mortes basin, Brazil: generating subsidies for preservation and conservation of this critical natural resource
}

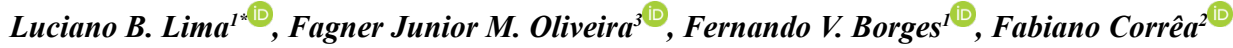 \\ \& Dilermando P. Lima-Junior ${ }^{1,2}$ \\ ${ }^{1}$ Universidade Federal de Mato Grosso, Laboratório de Ecologia e Conservação de Ecossistemas Aquáticos, \\ Av. Universitária, 3.500, Setor Universitário, CEP 78.698-000, Pontal do Araguaia, MT, Brasil. \\ ${ }^{2}$ Universidade do Estado de Mato Grosso, Programa de Pós-Gradudação em Ecologia e Conservação, Campus \\ Nova Xavantina, Rua Prof. Dr. Renato Figueiro Varella, Brasil, Caixa Postal 08, CEP 78690-000, Nova \\ Xavantina, MT, Brasil. \\ ${ }^{3}$ Universidade Estadual de Maringá, Programa de Pós-Graduação em Ecologia de Ecossistemas Aquáticos \\ Continentais, Avenida Colombo no 5790, 87020-900, Maringá, PR, Brasil. \\ *Corresponding author: lucianobeneditolima@gmail.com
}

LIMA, L.B., OLIVEIRA, F.J.M., BORGES, F.V., CORRÊA, F., LIMA-JUNIOR, D.P. Streams fish from Upper Araguaia and Middle Rio da Mortes basin, Brazil: generating subsidies for preservation and conservation of this critical natural resource. Biota Neotropica 21(4): e20211205. https://doi.org/10.1590/1676-0611-BN-2021-1205

\begin{abstract}
The Araguaia River basin has the highest fish biodiversity within the Cerrado biome (Brazilian savannah), with many endemic and threatened species by human activities. Despite growing efforts to catalog Neotropical freshwater fish biodiversity, many regions are still undersampled. Our objective is to complement the information about stream fish in two hydrographic basins in the Cerrado. We sampled 72 streams with $50 \mathrm{~m}$ stretch in the Upper Araguaia $(\mathrm{n}=32)$ and Middle Rio das Mortes $(\mathrm{n}=40)$ basins. We collected 14,887 individuals distributed in 137 species, 81 genera, 30 families, and six orders. Characidae, Loricariidae, and Cichlidae were the families richer in species. We found a high diversity of rare fish species in the streams sampled, ca. $71.5 \%$ of the species had at least five individuals collected, and 18 species had only one collected specimen. The most frequent species were Astyanax cf. goyacensis, Knodus $\mathrm{cf}$. breviceps, and Characidium cf. zebra. Both basins shared around $43 \%$ of the species. We caught 76 species in Upper Araguaia and 120 species in Middle Rio das Mortes. Seventeen exclusive fish species occurred in Upper Araguaia, whereas 61 were found in the Middle Rio das Mortes basin. Our analysis showed lower diversity of fish in Upper Araguaia than in the Middle Rio das Mortes basin. Considering the exclusive fish species of both basins, the human threats in those regions, and the few existent protected areas, we need a better look at the aquatic biodiversity conservation of this ecosystem. Keywords: Biodiversity; Cerrado; Freshwater; Neotropical fishes; Headwaters.
\end{abstract}

\section{Peixes de riachos das bacias do alto rio Araguaia e médio Rio das Mortes, Brasil: gerando subsídios para preservação e conservação desse importante recurso natural}

Resumo: Abacia do rio Araguaia apresenta a maior diversidade de peixes no bioma Cerrado, muitas dessas são endêmicas e ameaçadas pelas atividades antropogênicas. Apesar dos crescentes esforços para catalogar a diversidade de peixes neotropicais muitas regiões ainda permanecem subamostradas. O objetivo do nosso estudo é complementar a informação sobre peixes de riachos para duas importantes bacias hidrográficas no Cerrado. Amostramos um total de 72 trechos de 50 metros em riachos nas bacias alto rio Araguaia $(n=32)$ e médio Rio das Mortes $(n=40)$. Coletamos um total de 14.887 indivíduos distribuídos em 137 espécies, 81 gêneros, 30 famílias e seis ordens. As famílias Characidae, Loricariidae e Cichlidae foram as tiveram maior número de espécies. Encontramos uma grande raridade de espécies de peixes nos riachos amostrados, cerca de $71,5 \%$ das espécies apresentaram ao menos até cinco indivíduos coletados e 18 espécies tiveram apenas um único exemplar. As espécies com maior ocorrência nos riachos foram, Astyanax cf. goyacensis Knodus cf. breviceps e Characidium cf. zebra. As duas bacias compartilham aproximadamente $43 \%$ das espécies de peixes coletadas. Encontramos 76 espécies para o alto rio Araguaia e 120 espécies para médio Rio das Mortes. Observamos 17 espécies exclusivas para a bacia do alto rio Araguaia e 61 espécies exclusivas para bacia do Médio Rio das Mortes. Nossas análises mostraram que a diversidade de peixes é menor na bacia do alto rio Araguaia quando comparada a bacia do médio Rio das Mortes. Considerando as ameaças antrópicas, o baixo número unidades de conservação e o elevado número de espécies exclusivas presentes em ambas as bacias, existe uma necessidade urgente concentrar esforços na conservação desses ecossistemas.

Palavras-chave: Biodiversidade; Cerrado; Água doce; Peixes Neotropicais; Riachos de Cabeceiras. 


\section{Introduction}

The Cerrado is a Brazilian biome considered one of the global biodiversity hotspots due to its high species riches, endemic rates, and human threats to its biodiversity (Myers et al. 2000). The origins of large South American rivers (Amazonica, Tocantins-Araguaia, Paraná, and São Francisco basin) are inserted into this biome, which is informally entitled the Brazilian "berço das águas" (water cradle). The water bodies of Cerrado (e.g., rivers, lakes, and streams) harbor about 1200 cataloged fish species (ICMBio 2020), corresponding to $25 \%$ of South American freshwater fish species. It is important to highlight that many of the Cerrado fishes are endemic and represent more than $42 \%(n=$ 131) of the threatened Brazilian fish species (Latrubesse et al. 2019). Among the river basins originating within the Cerrado, the Araguaia River basin harbors more considerable fish diversity (Dagosta et al. 2020), with more than 320 currently described species and many with a restricted distribution that mainly occupies the headwaters (first to third order) (Latrubesse et al. 2019). Unfortunately, the Araguaia River basin landscape is undergoing a rapid transformation. Agriculture plantations or pastures replace native vegetation, and water bodies are being dammed for hydropower dam construction and agricultural irrigation intensification (Coe et al. 2011, Latrubesse et al. 2019).

The high number of fish species with restricted distribution and the human-caused environmental degradation in these basins are significant challenges for fish biodiversity conservation (Nogueira et al. 2010, Latrubesse et al. 2019, Dagosta et al. 2020). Therefore, actions addressed to preserve this biodiversity should consider the wide variation in fish community composition among the catchment systems since many of these restricted-distribution fish species are exclusive from streams (Lima 2019).

Stream fish are among the most threatened aquatic organisms (Nogueira et al. 2010, Castro 1999, Castro \& Polaz 2020), and one of their main threats concerns habitat degradation (Barletta et al. 2010, Castro \& Polaz 2020). The riparian vegetation removal (i.e., the watercourse adjacent buffer zone) is the leading habitat homogenization cause (Casatti, Ferreira \& Carvalho 2009, Teresa \& Casatti 2012, Zeni et al. 2019). Consequently, the resulting eutrophication and silting of the river channel (Teresa \& Casatti 2012) also contribute to biodiversity homogenization. Besides habitat change, the streams fish suffer other kinds of threats, such as introducing non-native species, highway and dam construction, agricultural pesticides, and fertilizer intensification (Winemiller et al. 2008, Reid et al. 2019).

Unfortunately, scientific ecological investigations advance more slowly than changes in natural ecosystems. Thus, many species can be extinct, even before they are formally described by science. Besides, there is data scarcity to fill distinct information gaps for many organisms (Hortal et al. 2015) regarding species proper identification (Linnean shortfalls) and the spatial distribution of the species (Wallacean shortfalls). It is essential to highlight that the Linnean shortfall is the most significant data gap to be solved because it directly affects all other biodiversity knowledge gaps (for more details, see Hortal et al. 2015). Furthermore, due to the growing and constant threat to streams, actions seeking to synthesize information about these ecosystems to guide research and conservation measures are needed (Dudgeon et al. 2006, Barletta et al. 2010, Cetra et al. 2020). Thus, our goal was to complement the information about stream fish in two important hydrographic basins in the Cerrado biome.

\section{Materials and Methods}

\section{Study Area}

We sampled a total of 72 streams ranging from first to fourth order (scale 1:10000 IBGE) according to Strahler's (1957) classification in the Upper Araguaia and Middle Rio das Mortes basin, belonging to the Tocantins-Araguaia ecoregion (Figure 1, Table 1). The Araguaia River is a major fluvial system of the Cerrado, draining $375,000 \mathrm{~km}^{2}$ and with an average annual streamflow of $6,500 \mathrm{~m}^{3} \cdot \mathrm{s}^{-1}$ (Morais et al. 2008, Latrubesse et al. 2009). The Araguaia River sources in slopes of the "Caiapós" mountain, on the boundaries of Goiás and Mato Grosso states, at $850 \mathrm{~m}$ elevation above sea level and travels $2110 \mathrm{~km}$ up to its confluence with the Tocantins River. Thus, the Araguaia basin can be divided into three stretches: upper, middle, and lower (for more details about division, see Latrubesse \& Stevaux 2002, Aquino et al. 2010). The Upper Araguaia River stretch, where a portion of our sampling sites are found, has an approximate extension of $450 \mathrm{~km}$ and is located between its source and the "Registro do Araguaia" district, draining an approximate area comprising $375,000 \mathrm{~km}^{2}$ (Latrubesse \& Stevaux 2002, Latrubesse et al. 2009).

The Rio das Mortes source is near to the "São Jerônimo" mountain, in the Mid-Southern Mato Grosso region at $808 \mathrm{~m}$ above sea level, flowing the northwest direction of the state for approximately 1,070 $\mathrm{km}$ until it flows into the middle section of the Araguaia River, near of São Félix do Araguaia city. The Rio das Mortes is the main tributary river of the Araguaia River basin, with a drainage area of approximately $61,684 \mathrm{~km}^{2}$ and an average annual flow of roughly $891.53 \mathrm{~m}^{3} \cdot \mathrm{s}^{-1}$ (ANA 2020). The initial portion basin is inserted into a landscape with intensive agriculture activity, whereas the native vegetation is restricted to the hydrographic network margins. While in the middle stretch, where a portion of our sampling sites are located, the mainland use activity is livestock. However, due to indigenous lands (TIs) and the sharp slope of relief at the edges of the "Alcantilados" plateau, it is still possible to find relatively sizable native vegetation areas in this stretch (Lima 2009).

Our study area is localized in the upper and middle stretches of the Araguaia and Rio das Mortes basins, respectively. The climate of the region is $A w$, according to the Köppen classification (Alvares et al. 2013), with two seasonal periods: (i) rainy from October to April and (ii) dry from May to September. The annual mean precipitation ranges from 1200 to $1900 \mathrm{~mm}$, and the yearly average temperature is approximately $24{ }^{\circ} \mathrm{C}$, with higher temperatures in the rainy period (INMET 2020).

\section{Data Sampling}

Using geography information systems (GIS) tools, we chose 32 streams in the Araguaia River and 40 in the Rio das Mortes basin, totaling 72 stretches of streams to be sampled. The sites were selected based on the independence between them and accessibility criteria. We used the collection sampling method modified from the Biodiversity Research Program (PPBIO), which consisted of sampling a 50-meter stream stretch (Mendonça et al. 2005). We collected in each stream environmental variables related to the limnological conditions and structural variables related to the environmental characterization of the streams. We measured the limnological conditions (i.e., conductivity, dissolved oxygen, $\mathrm{pH}$, turbidity, and water temperature) of the streams only once at the beginning of the sample stretch using a portable multiparametric probe (Horiba U-50). We divided each sampled 


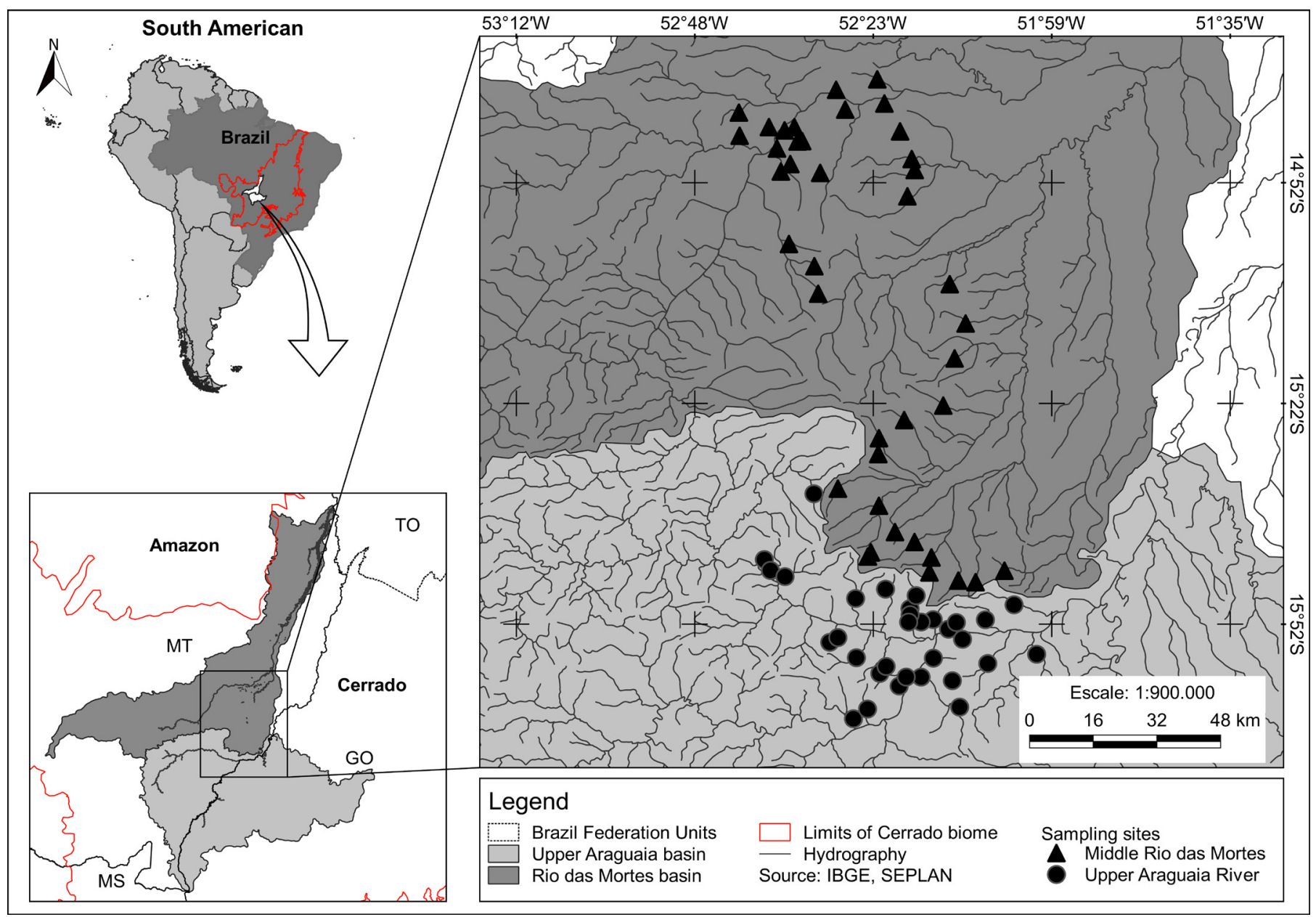

Figure 1. Localization of 72 streams sampled in the Upper Araguaia and Middle Rio das Mortes basin, Tocantins-Araguaia ecoregion.

stretch-50 m into six equidistant transects and recorded the following structural variables, representing the average values of each one of the measured variables in each transect: width, depth obtained from five measurements from one margin to the other, surface water velocity using the method fluctuate material (Teresa \& Casatti 2012), and the proportion of substrate structure (i.e., sand, gravel, pebbles, rock, slab, and silt). In addition, we visually quantified the presence of trunks, leaf-litter, and margin structure variables (i.e., thin roots, thick roots, and grass banks) (Cummins 1974, Teresa \& Casatti 2012).

We sampled the fish of the streams during the dry periods (May to September) between 2014 - 2017 for more catching efficiency and reduced seasonality effects on collections (Ueida \& Castro 1999, Pease et al. 2012). Before initializing the sampling, we blocked the sample stretch limits with 5-mm mesh nets to prevent fish escape. Then, we sampled the fish through the standardized active collection, which consisted of employing four people for approximately one hour. Later, we sampled 35 streams stretch during the day using seine nets $(3.0 \times 1.5 \mathrm{~m} \times 5.0 \mathrm{~mm}$ mesh $)$ and dip nets $(0.5 \times 0.45 \mathrm{~m} \times 5.0$ $\mathrm{mm}$ mesh). Next, we used the electrofishing method (Honda EG1000 generator $-220 \mathrm{~V}, \mathrm{CA}$ ) with a single passage downstream, upstream, lasting approximately one hour to sample the other 37 streams. After the sampling, the individuals were anesthetized with benzocaine and sacrificed according to the Federal Council of Veterinary Medicine (in Portuguese CFMV 2012). In the laboratory, we measured the standard length $(\mathrm{cm})$, weighted $(\mathrm{g})$, and identified all specimens collected until the lowest taxonomic level possible using specialized bibliography (Venere \& Garutti 2011), and to elaborate the taxonomic list using the Catalogue of Fishes (Fricke et al. 2021).

The Instituto Chico Mendes de Conservação da Biodiversidade(ICMBio; Permit No. 45316-1) and the Animal Use Ethics Committee of the Universidade Federal de Mato Grosso (CEUA/ UFMT $-\mathrm{N}^{\circ}$. 23108.152116) authorized our field collections. We stored the fish collected in the Laboratório de Ecologia e Conservação de Ecossistemas Aquáticos at the Universidade Federal de Mato Grosso, Campus Araguaia, Pontal do Araguaia, Mato Grosso.

\section{Data Analysis}

We used a principal component analysis (PCA) to summarize the environmental characteristics of the streams. After running the PCA, we standardized all environmental variables (except $\mathrm{pH}$ ) to zero mean and unit variances ( $\mathrm{z}$ transformation). Next, we analyzed the richness values (number of species) and abundance (total number of individuals by species) of the fish communities using descriptive statistics. We considered as exclusive species those that occurred only within one basin and as unique species those that occurred only in one stream reach, and singletons those with only one specimen (Novotný \& Basset 2000). Finally, we evaluated the species richness differences and the efficiency of the sampling effort within both basins using the rarefaction 
Table 1. The list of sampled sites in the Upper Araguaia River (UAR) and Middle Rio das Mortes (MRM) basin, Tocantins-Araguaia ecoregion.

\begin{tabular}{|c|c|c|c|c|c|c|c|c|}
\hline Sites & Name & Latitude & Longitude & Order & $\begin{array}{c}\text { Width } \\
\text { (m) }\end{array}$ & $\begin{array}{c}\text { Depth } \\
\text { (cm) }\end{array}$ & Collection method & Elevation \\
\hline MRM01 & Salgadinho stream & $14^{\circ} 40^{\prime} 46.20^{\prime \prime} \mathrm{S}$ & $52^{\circ} 21^{\prime} 53.64^{\prime \prime} \mathrm{W}$ & Third & 4.04 & 15.78 & Electrofishing & 263 \\
\hline MRM02 & Unnamed stream & $14^{\circ} 46^{\prime} 54.48^{\prime \prime} \mathrm{S}$ & $52^{\circ} 36^{\prime} 26.64^{\prime \prime} \mathrm{W}$ & Second & 2.95 & 15.52 & Electrofishing & 279 \\
\hline MRM03 & Queixada stream & $14^{\circ} 44^{\prime} 25.80^{\prime \prime} \mathrm{S}$ & $52^{\circ} 35^{\prime} 25.08^{\prime \prime} \mathrm{W}$ & Second & 3.55 & 36.39 & Electrofishing & 273 \\
\hline MRM04 & Unnamed stream & $14^{\circ} 43^{\prime} 59.52^{\prime \prime} \mathrm{S}$ & $52^{\circ} 34^{\prime} 5.16^{\prime \prime} \mathrm{W}$ & Second & 12.81 & 21.72 & Electrofishing & 276 \\
\hline MRM05 & Voadeira stream & $14^{\circ} 41^{\prime} 35.88^{\prime \prime} \mathrm{S}$ & $52^{\circ} 27^{\prime} 11.88^{\prime \prime} \mathrm{W}$ & Third & 7.23 & 19.58 & Electrofishing & 269 \\
\hline MRM06 & Unnamed stream & $14^{\circ} 45^{\prime} 9.36^{\prime \prime S}$ & $52^{\circ} 41^{\prime} 30.48^{\prime \prime} \mathrm{W}$ & Second & 2.28 & 33.65 & Electrofishing & 322 \\
\hline MRM07 & Unnamed stream & $14^{\circ} 43^{\prime} 58.80^{\prime \prime} \mathrm{S}$ & $52^{\circ} 37^{\prime} 32.16^{\prime \prime} \mathrm{W}$ & Third & 3.72 & 21.12 & Electrofishing & 295 \\
\hline MRM08 & Barreira stream & $14^{\circ} 38^{\prime} 53.16^{\prime \prime} \mathrm{S}$ & $52^{\circ} 28^{\prime} 26.40^{\prime \prime} \mathrm{W}$ & Third & 2.80 & 15.68 & Electrofishing & 277 \\
\hline MRM09 & Affluent of the Piau stream & $14^{\circ} 42^{\prime} 0.36^{\prime \prime} \mathrm{S}$ & $52^{\circ} 41^{\prime} 36.24 ” \mathrm{~W}$ & Third & 2.11 & 39.65 & Electrofishing & 334 \\
\hline MRM10 & Bacaba stream & $14^{\circ} 53^{\prime} 26.16^{\prime \prime} \mathrm{S}$ & $52^{\circ} 18^{\prime} 46.44^{\prime \prime} \mathrm{W}$ & Third & 3.62 & 22.18 & Electrofishing & 298 \\
\hline MRM11 & Cachoeirinha stream & $14^{\circ} 49^{\prime} 50.88^{\prime \prime S} \mathrm{~S}$ & $52^{\circ} 17^{\prime} 47.40^{\prime \prime} \mathrm{W}$ & Third & 3.67 & 32.28 & Electrofishing & 288 \\
\hline MRM12 & Duas Pontes stream & $14^{\circ} 48^{\prime} 20.88^{\prime \prime} \mathrm{S}$ & $52^{\circ} 18^{\prime} 11.52^{\prime \prime} \mathrm{W}$ & Fourth & 5.66 & 35.38 & Electrofishing & 285 \\
\hline MRM13 & Estilac stream & $14^{\circ} 37^{\prime} 27.84$ ”S & $52^{\circ} 22^{\prime} 51.60^{\prime \prime} \mathrm{W}$ & Second & 3.18 & 30.53 & Electrofishing & 298 \\
\hline MRM14 & Duas Pontes stream & $15^{\circ} 5^{\prime} 24.72 ” S$ & $52^{\circ} 13^{\prime} 2.64^{\prime \prime} \mathrm{W}$ & Fourth & 7.60 & 29.48 & Electrofishing & 301 \\
\hline MRM15 & Pindaibinha stream & $15^{\circ} 10^{\prime} 46.92$ ”S & $52^{\circ} 10^{\prime} 53.40^{\prime \prime} \mathrm{W}$ & Fourth & 3.72 & 32.37 & Electrofishing & 315 \\
\hline UAR01 & Avoadeira stream & $15^{\circ} 51^{\prime} 13.32$ ”S & $52^{\circ} 15^{\prime} 17.28^{\prime \prime} \mathrm{W}$ & Third & 3.33 & 30.60 & Hand net and Dip net & 521 \\
\hline UAR02 & Peixinho stream & $15^{\circ} 51^{\prime} 32.76$ ”S & $52^{\circ} 16^{\prime} 55.20^{\prime \prime} \mathrm{W}$ & First & 3.25 & 13.62 & Hand net and Dip net & 373 \\
\hline UAR03 & Pitomba stream & $15^{\circ} 51 ' 14.76^{\prime \prime} \mathrm{S}$ & $52^{\circ} 8,12.48^{\prime \prime} \mathrm{W}$ & Third & 3.32 & 12.48 & Hand net and Dip net & 322 \\
\hline UAR04 & Ouro Fino stream & $15^{\circ} 49^{\prime} 12.00^{\prime \prime} \mathrm{S}$ & $52^{\circ} 4 ' 19.56^{\prime \prime} \mathrm{W}$ & Third & 3.93 & 38.14 & Hand net and Dip net & 335 \\
\hline UAR05 & Águas Quentes stream & $15^{\circ} 52^{\prime} 31.80^{\prime \prime} \mathrm{S}$ & $52^{\circ} 13^{\prime} 12.36^{\prime \prime} \mathrm{W}$ & First & 3.32 & 12.88 & Hand net and Dip net & 325 \\
\hline MRM16 & Areia stream & $15^{\circ} 44^{\prime} 45.24 ” \mathrm{~S}$ & $52^{\circ} 15^{\prime} 47.52^{\prime \prime} \mathrm{W}$ & First & 10.61 & 23.25 & Hand net and Dip net & 396 \\
\hline UAR06 & Lontra stream & $15^{\circ} 49^{\prime} 43.32^{\prime \prime} \mathrm{S}$ & $52^{\circ} 18^{\prime} 25.56^{\prime \prime} \mathrm{W}$ & First & 1.99 & 12.56 & Hand net and Dip net & 349 \\
\hline UAR07 & Unnamed stream & $15^{\circ} 50^{\prime} 33.3^{\prime \prime S}$ & $52^{\circ} 18^{\prime} 24.9 ’ \mathrm{~W}$ & First & 6.31 & 9.36 & Hand net and Dip net & 345 \\
\hline UAR08 & Unnamed stream & $15^{\circ} 51^{\prime} 33.48^{\prime \prime} \mathrm{S}$ & $52^{\circ} 18^{\prime} 37.08^{\prime \prime} \mathrm{W}$ & First & 1.99 & 7.17 & Hand net and Dip net & 355 \\
\hline MRM17 & Grande stream & $15^{\circ} 44^{\prime} 31.20^{\prime \prime} \mathrm{S}$ & $52^{\circ} 5,37.68^{\prime \prime} \mathrm{W}$ & Third & 2.90 & 45.33 & Hand net and Dip net & 348 \\
\hline MRM18 & Grande stream & $15^{\circ} 35^{\prime} 39.48^{\prime \prime} \mathrm{S}$ & $52^{\circ} 22^{\prime} 38.28^{\prime \prime} \mathrm{W}$ & Fourth & 7.92 & 32.57 & Hand net and Dip net & 355 \\
\hline MRM19 & Cava Funda stream & $15^{\circ} 39^{\prime} 14.76^{\prime \prime} \mathrm{S}$ & $52^{\circ} 20^{\prime} 27.96^{\prime \prime} \mathrm{W}$ & Third & 5.01 & 19.20 & Hand net and Dip net & 398 \\
\hline MRM20 & Taquaral stream & $15^{\circ} 40^{\prime} 36.48^{\prime \prime S}$ & $52^{\circ} 17^{\prime} 48.84^{\prime \prime} \mathrm{W}$ & Third & 7.77 & 43.92 & Hand net and Dip net & 369 \\
\hline MRM21 & Fogaça stream & $15^{\circ} 46^{\prime} 4.80^{\prime \prime} \mathrm{S}$ & $52^{\circ} 9^{\prime} 32.40 ” \mathrm{~W}$ & Third & 2.66 & 9.30 & Hand net and Dip net & 397 \\
\hline MRM22 & Ínsula stream & $15^{\circ} 45^{\prime} 52.20 ” \mathrm{~S}$ & $52^{\circ} 11^{\prime} 52.80^{\prime \prime} \mathrm{W}$ & Third & 3.68 & 13.27 & Hand net and Dip net & 388 \\
\hline MRM23 & Taquaralzinho stream & $15^{\circ} 42^{\prime} 42.48^{\prime \prime S}$ & $52^{\circ} 15^{\prime} 31.32^{\prime \prime} \mathrm{W}$ & Third & 4.53 & 37.62 & Hand net and Dip net & 369 \\
\hline UAR09 & Fundo stream & $15^{\circ} 47^{\prime} 56.76^{\prime \prime} \mathrm{S}$ & $52^{\circ} 17^{\prime} 35.52^{\prime \prime} \mathrm{W}$ & Fourth & 5.00 & 21.82 & Hand net and Dip net & 343 \\
\hline UAR10 & Portão stream & $15^{\circ} 34^{\prime} 2.64 ” S$ & $52^{\circ} 31^{\prime} 26.76^{\prime \prime} \mathrm{W}$ & Second & 4.98 & 27.57 & Hand net and Dip net & 427 \\
\hline MR24 & Capa stream & $15^{\circ} 33^{\prime} 19.44^{\prime \prime} \mathrm{S}$ & $52^{\circ} 28^{\prime} 11.64^{\prime \prime} \mathrm{W}$ & Fourth & 4.60 & 51.30 & Hand net and Dip net & 421 \\
\hline UAR11 & Cambaúva stream & $15^{\circ} 43^{\prime} 1.92 ” S$ & $52^{\circ} 38^{\prime} 9.60 ’ \mathrm{~W}$ & First & 3.69 & 56.60 & Hand net and Dip net & 387 \\
\hline UAR12 & Índios stream & $15^{\circ} 44^{\prime} 30.84$ ”S & $52^{\circ} 37^{\prime} 20.28^{\prime \prime} \mathrm{W}$ & Third & 7.81 & 31.10 & Hand net and Dip net & 326 \\
\hline UAR13 & Affluent of the Bateia stream & $15^{\circ} 45^{\prime} 21.24 ” \mathrm{~S}$ & $52^{\circ} 35^{\prime} 21.84^{\prime \prime} \mathrm{W}$ & First & 2.61 & 17.15 & Hand net and Dip net & 337 \\
\hline UAR14 & Ponte Queimada stream & $15^{\circ} 48^{\prime} 20.52 ” \mathrm{~S}$ & $52^{\circ} 25^{\prime} 47.64^{\prime \prime} \mathrm{W}$ & Fourth & 5.99 & 30.70 & Hand net and Dip net & 377 \\
\hline UAR15 & $\begin{array}{c}\text { Affluent of the Monte Negro } \\
\text { stream }\end{array}$ & $15^{\circ} 58^{\prime} 33.96 ” \mathrm{~S}$ & $52^{\circ} 22^{\prime} 30.72^{\prime} \mathrm{W}$ & First & 1.51 & 22.97 & Hand net and Dip net & 379 \\
\hline UAR16 & Buritirana stream & $15^{\circ} 57^{\prime} 37.08^{\prime \prime} \mathrm{S}$ & $52^{\circ} 21^{\prime} 39.60^{\prime \prime} \mathrm{W}$ & First & 2.14 & 27.70 & Hand net and Dip net & 360 \\
\hline UAR17 & Marimbondo stream & $15^{\circ} 54^{\prime} 19.08^{\prime \prime} \mathrm{S}$ & $52^{\circ} 29^{\prime} 17.88^{\prime \prime} \mathrm{W}$ & Second & 3.03 & 41.00 & Hand net and Dip net & 344 \\
\hline UAR18 & Mangabeira stream & $15^{\circ} 53^{\prime} 40.56 " \mathrm{~S}$ & $52^{\circ} 28^{\prime} 12.00^{\prime \prime} \mathrm{W}$ & Third & 2.18 & 39.30 & Hand net and Dip net & 326 \\
\hline UAR19 & Babilônia stream & $15^{\circ} 56^{\prime} 25.44^{\prime \prime S}$ & $52^{\circ} 25^{\prime} 41.16^{\prime \prime} \mathrm{W}$ & Fourth & 4.49 & 30.93 & Hand net and Dip net & 330 \\
\hline UAR20 & Buritirana stream & $5^{\circ} 51^{\prime} 36.72 ” S$ & $52^{\circ} 12^{\prime} 8.64 ” \mathrm{~W}$ & Second & 6.37 & 31.27 & Hand net and Dip net & 338 \\
\hline UAR21 & Ribeirão das Mulas stream & $15^{\circ} 55^{\prime} 57.00^{\prime \prime} \mathrm{S}$ & $52^{\circ} 11^{\prime} 15.60^{\prime \prime} \mathrm{W}$ & Fourth & 5.23 & 20.57 & Hand net and Dip net & 299 \\
\hline UAR22 & Ribeirão Capivara stream & $15^{\circ} 57^{\prime} 12.60$ ”'S & $52^{\circ} 7^{\prime} 53.40^{\prime \prime} \mathrm{W}$ & Fourth & 3.99 & 29.80 & Hand net and Dip net & 319 \\
\hline UAR23 & $\begin{array}{c}\text { Affluent of the Avoadeira } \\
\text { stream }\end{array}$ & $15^{\circ} 47^{\prime} 3.84^{\prime \prime S}$ & $52^{\circ} 21^{\prime} 44.64^{\prime \prime} \mathrm{W}$ & Second & 2.70 & 27.57 & Hand net and Dip net & 360 \\
\hline
\end{tabular}




\begin{tabular}{|c|c|c|c|c|c|c|c|c|}
\hline UAR24 & Grande stream & $15^{\circ} 53^{\prime} 54.24 ’ \mathrm{~S}$ & $52^{\circ} 11^{\prime} 18.24$ 'W & Third & 5.07 & 14.40 & Hand net and Dip net & 302 \\
\hline UAR25 & Areia stream & $15^{\circ} 59^{\prime} 4.20^{\prime \prime} \mathrm{S}$ & $52^{\circ} 16^{\prime} 55.20^{\prime \prime} \mathrm{W}$ & Fourth & 6.33 & 26.80 & Hand net and Dip net & 300 \\
\hline UAR26 & Jaraguá stream & $15^{\circ} 56^{\prime} 29.04 ” S$ & $52^{\circ} 15^{\prime} 15.48^{\prime \prime} \mathrm{W}$ & Third & 3.52 & 10.67 & Hand net and Dip net & 303 \\
\hline MRM25 & Sucuri stream & $15^{\circ} 15^{\prime} 33.12^{\prime \prime S}$ & $52^{\circ} 12^{\prime} 23.04^{\prime \prime} \mathrm{W}$ & Third & 4.20 & 35.35 & Electrofishing & 338 \\
\hline MRM26 & Matrinxã stream & $15^{\circ} 21$ '59.04"S & $52^{\circ} 13^{\prime} 55.20^{\prime \prime} \mathrm{W}$ & Third & 5.04 & 38.30 & Electrofishing & 363 \\
\hline MRM27 & Água Limpa stream & $15^{\circ} 23^{\prime} 57.84^{\prime \prime} \mathrm{S}$ & $52^{\circ} 19^{\prime} 12.72^{\prime \prime} \mathrm{W}$ & Third & 4.63 & 22.47 & Electrofishing & 415 \\
\hline MRM28 & Mineiro stream & $15^{\circ} 26^{\prime} 26.16^{\prime \prime} \mathrm{S}$ & $52^{\circ} 22^{\prime} 37.92^{\prime \prime} \mathrm{W}$ & Third & 2.96 & 22.27 & Electrofishing & 413 \\
\hline MRM29 & Papagaio stream & $15^{\circ} 28^{\prime} 35.76^{\prime \prime} \mathrm{S}$ & $52^{\circ} 22^{\prime} 43.32^{\prime \prime} \mathrm{W}$ & Fourth & 6.08 & 22.28 & Electrofishing & 388 \\
\hline UAR27 & Caixão stream & $16^{\circ} 4$ '45.12”S & $52^{\circ} 26^{\prime} 4.92^{\prime \prime} \mathrm{W}$ & Fourth & 3.05 & 24.05 & Electrofishing & 320 \\
\hline UAR28 & Cambaúva stream & $16^{\circ} 3^{\prime} 25.56^{\prime \prime} \mathrm{S}$ & $52^{\circ} 24^{\prime} 10.80^{\prime \prime} \mathrm{W}$ & Fourth & 1.94 & 11.60 & Electrofishing & 336 \\
\hline UAR29 & Cervo stream & $16^{\circ} 0,17.28^{\prime \prime} \mathrm{S}$ & $52^{\circ} 19^{\prime} 53.40^{\prime \prime} \mathrm{W}$ & Third & 2.15 & 22.54 & Electrofishing & 326 \\
\hline UAR30 & Grota Funda stream & $15^{\circ} 59^{\prime} 3.48^{\prime \prime S}$ & $52^{\circ} 18^{\prime} 56.52^{\prime \prime} \mathrm{W}$ & Third & 4.28 & 19.28 & Electrofishing & 332 \\
\hline MRM30 & Unnamed stream & $14^{\circ} 49^{\prime} 1.20^{\prime \prime S}$ & $52^{\circ} 34^{\prime} 38.64^{\prime \prime} \mathrm{W}$ & First & 2.03 & 17.73 & Electrofishing & 422 \\
\hline MRM31 & Unnamed stream & $14^{\circ} 45^{\prime} 56.88^{\prime \prime} \mathrm{S}$ & $52^{\circ} 33^{\prime} 39.60^{\prime \prime} \mathrm{W}$ & First & 1.05 & 41.20 & Electrofishing & 395 \\
\hline MRM32 & Unnamed stream & $14^{\circ} 45^{\prime} 53.28^{\prime \prime} \mathrm{S}$ & $52^{\circ} 33^{\prime} 1.80^{\prime \prime} \mathrm{W}$ & Second & 3.23 & 28.40 & Electrofishing & 425 \\
\hline MRM33 & Chupador stream & $14^{\circ} 50 ’ 13.92 ” \mathrm{~S}$ & $52^{\circ} 30^{\prime} 35.64^{\prime \prime} \mathrm{W}$ & Second & 1.83 & 27.33 & Electrofishing & 437 \\
\hline MRM34 & Unnamed stream & $14^{\circ} 44^{\prime} 34.08^{\prime \prime} \mathrm{S}$ & $52^{\circ} 19^{\prime} 46.20^{\prime \prime} \mathrm{W}$ & First & 6.93 & 9.57 & Electrofishing & 306 \\
\hline MRM35 & Unnamed stream & $15^{\circ} 6^{\prime} 42.84^{\prime \prime} \mathrm{S}$ & $52^{\circ} 30^{\prime} 51.84^{\prime \prime} \mathrm{W}$ & Second & 1.22 & 46.23 & Electrofishing & 616 \\
\hline MRM36 & Unnamed stream & $15^{\circ} 2 ' 58.20^{\prime \prime} \mathrm{S}$ & $52^{\circ} 31^{\prime} 22.80^{\prime \prime} \mathrm{W}$ & First & 1.32 & 41.80 & Electrofishing & 575 \\
\hline MRM37 & Unnamed stream & $14^{\circ} 59^{\prime} 56.40^{\prime \prime S}$ & $52^{\circ} 34^{\prime} 49.80^{\prime \prime} \mathrm{W}$ & Second & 2.10 & 65.68 & Electrofishing & 490 \\
\hline MRM38 & Unnamed stream & $14^{\circ} 50^{\prime} 2.76^{\prime \prime} \mathrm{S}$ & $52^{\circ} 35^{\prime} 55.32^{\prime \prime} \mathrm{W}$ & First & 1.71 & 30.92 & Electrofishing & 353 \\
\hline MRM39 & Unnamed stream & $15^{\circ} 42 ’ 34.92 ” S$ & $52^{\circ} 24^{\prime} 7.92^{\prime \prime} \mathrm{W}$ & First & 1.34 & 10.55 & Electrofishing & 719 \\
\hline MRM40 & Unnamed stream & $15^{\circ} 42^{\prime} 1.08^{\prime \prime S}$ & $52^{\circ} 23^{\prime} 43.44^{\prime \prime} \mathrm{W}$ & First & 1.30 & 16.35 & Electrofishing & 671 \\
\hline UAR31 & Volta stream & $16^{\circ} 3$ '9.72”S & $52^{\circ} 11^{\prime} 41.64^{\prime \prime} \mathrm{W}$ & Second & 2.72 & 29.97 & Electrofishing & 339 \\
\hline UAR32 & Unnamed stream & 1559'33.36”S & $52^{\circ} 12^{\prime} 41.40^{\prime \prime} \mathrm{W}$ & First & 1.03 & 11.68 & Electrofishing & 356 \\
\hline
\end{tabular}

and extrapolation (R/E) method (Colwell et al. 2012). We based our analyses on incidence data derived from the Hill number series with a 95\% confidence interval obtained with the bootstrap method (Hill 1973, Chao et al. 2014, Colwell et al. 2012) using the $i N E X T$ function from the $i N E X T$ package (Hsieh et al. 2016). We performed all analyses and descriptive statistics with $R$ software version 3.6.1 (R Core Team 2019). We used the vegan package (Oksanen et al. 2018) to perform PCA and package ggplot2 (Wickham 2009) to visualize the results.

\section{Results}

In general, the studied streams had low conductivity water (mean $=59.79 \mu \mathrm{S} / \mathrm{cm})$, high dissolved oxygen levels $($ mean $=8.02 \mathrm{mg} / \mathrm{l})$, slightly acidic $($ mean $=5.87 \mathrm{pH})$, low turbidity $($ mean $=2.38 \mathrm{NTU})$, and substrates with sand predominance (mean $=45.58 \%$ ) and gravel (mean $=16.95 \%$ ). The first two PCA axes explained $27.76 \%$ of the environmental variation features in the streams (Figure 2). The first axis explained $16.57 \%$ of the variation and was positively associated with water temperature, silt, and grass banks and negatively associated with trunks and leaf-litters (Figure 2). The second axis accounted for $11.19 \%$ of the variation and was negatively associated with depth and sand (Figure 2). The first axis distinguished streams with a high proportion of grass banks in margins structures from streams with a high presence internal habits structure (Figure 2). The second axis differentiated streams with a higher proportion of pools and a higher proportion of unconsolidated substrates (sand) from streams with a high proportion of consolidated substrates (rocks) (Figure 2).

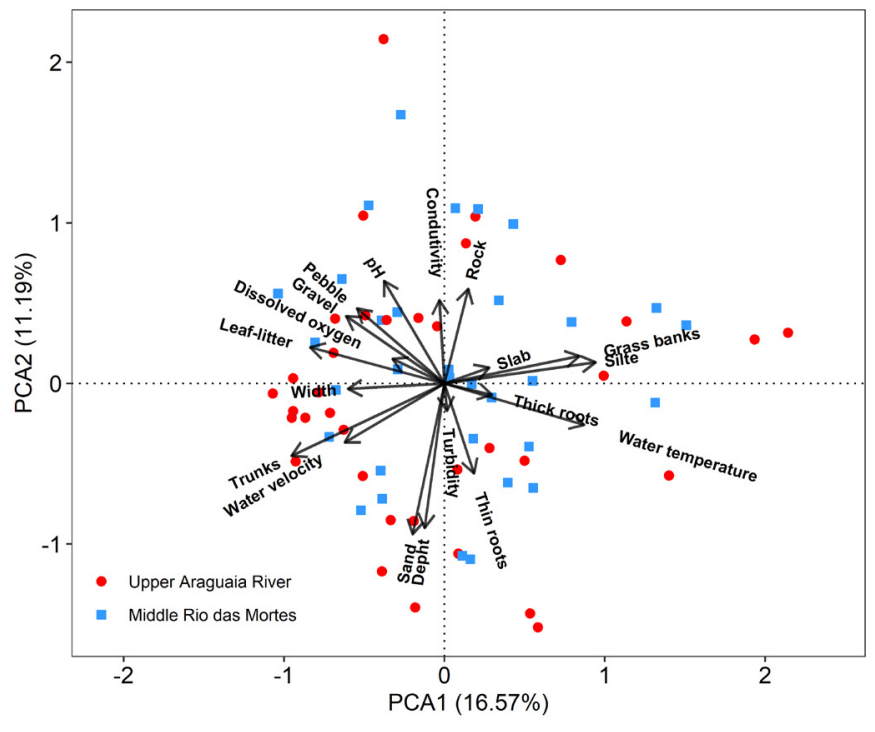

Figure 2. Biplot for principal components analysis (PCA) representing the main environmental variability in the sampling sites in the Upper Araguaia River and Middles Rio das Mortes basin, Tocantins-Araguaia ecoregion.

We collected a total of 14,887 individuals distributed in 137 species, 81 genera, 30 families, and six orders (Figure 3). The most speciesrich families were Characidae, Loricariidae, and Cichlidae (40, 25, and 12 species, respectively). More than half of the individuals we sampled were concentrated in eight species (Astyanax cf. goyacensis, Psalidodon xavante, Hyphessobrycon aff. tenuis, Knodus cf. breviceps, 


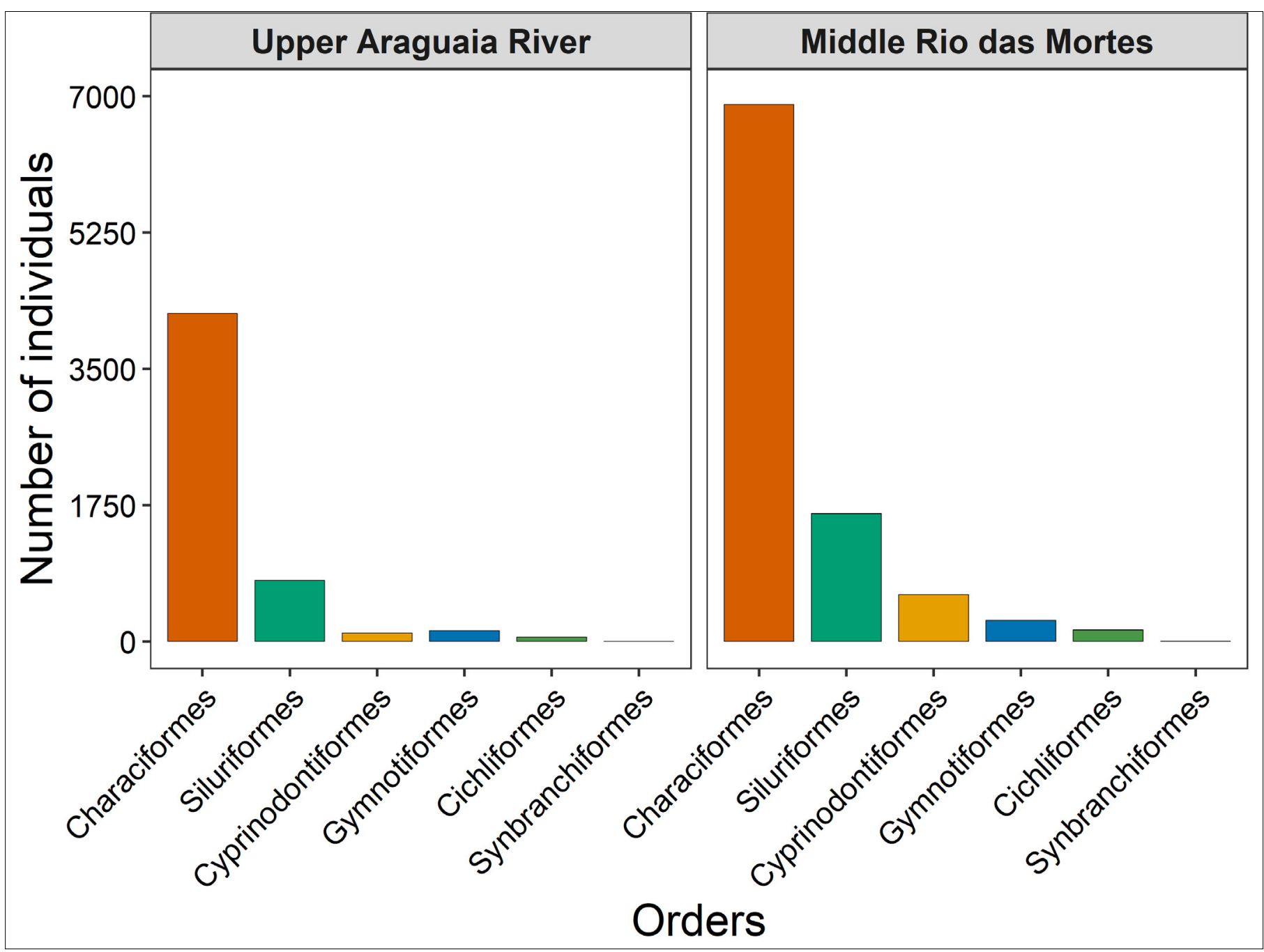

Figure 3. The individual abundance of six orders collected in the Upper Araguaia and Middle Rio das Mortes basins, Tocantins-Araguaia ecoregion.

Odontostilbe sp., Phenacogaster sp., Aspidoras poecilus, Melanorivulus zygonectes) (Table 2, Figure 4). The remaining 98 species $(71.5 \%)$ had at least five individuals collected, and 18 species were considered singletons. The average richness per stream was 15.5 species $(S D=$ 11). The species richness ranged from streams with only one species to streams with 48 species. The most frequent species in our sampled streams were Astyanax cf. goyacensis $(n=51)$, Knodus cf. breviceps $(n=43)$, Characidium cf. zebra $(n=40)$, Imparfinis mirini $(n=40)$, and Moenkhausia oligolepis $(n=40)$. We found 36 unique species, and 45 species identified only genera level. The Hypostomus and Ancistrus genera are the richer genera, showing the largest number of species identified up to the genera level.

The basins shared approximately $43 \%(S=59)$ of the collected species. When we analyzed the species richness by basin, we found 76 species for Upper Araguaia and 120 in the Middle Rio das Mortes basin. We found 17 exclusives to Upper Araguaia and 61 species exclusive to the Middle Rio das Mortes basin. The rarefaction analysis showed that fish diversity in both Hill diversity series was lower in the Upper Araguaia River compared to the Middle Rio das Mortes basin (Figure 5a). The sampling effort analysis showed that the species richness for both basins was well represented, with a percentage of sample coverage greater than $93 \%$ of the estimated species (Figure $5 \mathrm{bc}$ ).
We sampled more than 93\% of the Upper Rio Araguaia basin's estimated species richness, and 95\% of the Middle Rio das Mortes basin's estimated species richness (Figure 5c).

\section{Discussion}

The predominance of the orders and families found in our study aligns with the expected patterns for Neotropical fishes in streams (Castro 1999, Lowe-McConnell 1999, Winemiller et al. 2008, Castro $\&$ Polaz 2020). The species richness and abundance of Characiformes, Siluriformes, and Cichliformes recorded in our study also follow the pattern reported in other studies with fish streams to some basins in the Cerrado biome (Leal et al. 2014, Barbosa et al. 2019). This pattern can be explained by the dominance of those orders in the Neotropical region (Lowe-McConnell 1999, Albert et al. 2011, Reis et al. 2016). The predominance of the Characidae family in the studied streams could be because this group is the richest in the Characiformes order, containing over 550 species (Albert et al. 2011) and displaying an extraordinary variation in morphological forms, feeding behaviors, and reproductive strategies (Melo et al. 2004, Winemiller et al. 2008). Such variety allows this group of fish to occur in the most diverse aquatic habitats. In turn, the predominance of Loricariidae fish can be due to the high species 
Table 2. Species list with your respective abundance in Upper Araguaia River (UAR) and Middle Rio das Mortes (MRM), Tocantins-Araguaia ecoregion.

\begin{tabular}{|c|c|c|c|}
\hline \multirow[b]{2}{*}{ ORDER/Family/Species } & \multicolumn{2}{|c|}{ Basin } & \multirow[b]{2}{*}{ Total } \\
\hline & UAR & MRM & \\
\hline \multicolumn{4}{|l|}{ CHARACIFORMES } \\
\hline Apareiodon sp.1 & & 2 & 2 \\
\hline Apareiodon sp.2 & 88 & & 88 \\
\hline \multicolumn{4}{|l|}{ Curimatidae } \\
\hline Curimatella immaculata (Fernández-Yépez, 1948) & & 1 & 1 \\
\hline Cyphocharax gouldingi Vari, 1992 & 25 & 131 & 156 \\
\hline Steindachnerina amazonica (Steindachner, 1911) & 310 & 26 & 336 \\
\hline \multicolumn{4}{|l|}{ Prochilondotidae } \\
\hline Leporinus klausewitzi Géry, 1960 & 1 & 3 & 4 \\
\hline Leporinus sp.1 & 10 & 19 & 29 \\
\hline Leporinus venerei Britski \& Birindelli, 2008 & & 5 & 5 \\
\hline \multicolumn{4}{|l|}{ Erythrinidae } \\
\hline Hoplerythrinus unitaeniatus (Spix \& Agassiz, 1829) & & 32 & 32 \\
\hline Hoplias cf. malabaricus (Bloch, 1974) & 17 & 58 & 75 \\
\hline \multicolumn{4}{|l|}{ Lebiasinidae } \\
\hline Pyrrhulina australis Eigenmann \& Kennedy, 1903 & 5 & 15 & 20 \\
\hline Serrasalmus spilopleura Kner, 1858 & & 1 & 1 \\
\hline \multicolumn{4}{|l|}{ Characidae } \\
\hline Aphyocharax alburnus (Günther, 1869) & 5 & 2 & 7 \\
\hline Aphyocharax sp.1 & & 20 & 20 \\
\hline Astyanax argyrimarginatus Garutti, 1999 & 165 & & 165 \\
\hline Astyanax cf. goyacensis Eigenmann, 1908 & 482 & 601 & 1083 \\
\hline Astyanax elachylepis Bertaco \& Lucinda, 2005 & 15 & 124 & 139 \\
\hline Astyanax sp. & & 272 & 272 \\
\hline Bryconamericus novae Eigenmann \& Henn, 1914 & & 56 & 56 \\
\hline Creagrutus figueiredoi Vari \& Harold, 2001 & 39 & 61 & 100 \\
\hline Creagrutus menezesi Vari \& Harold, 2001 & 99 & & 99 \\
\hline Creagrutus seductus Vari \& Harold, 2001 & 70 & & 70 \\
\hline Hemigrammus aff. levis Durbin, 1908 & 6 & 8 & 14 \\
\hline Hemigrammus cf. rodwayi Durbin, 1909 & 253 & 132 & 385 \\
\hline Hyphessobrycon aff. tenuis Géry, 1964 & 220 & 403 & 623 \\
\hline Hyphessobrycon sp. & 261 & & 261 \\
\hline
\end{tabular}


...continuation

Jupiaba acanthogaster (Eigenmann, 1911)

18

Jupiaba elassonaktis Pereira \& Lucinda, 2007

Jupiaba polylepis (Günther, 1864)

Knodus cf. breviceps (Eigenmann, 1908)

Knodus sp.

Microschemobrycon sp.1

Microschemobrycon sp.2

Moenkhausia aurantia Bertaco, Jerep \& Carvalho, 2011

Moenkhausia cf. comma Eigenmann, 1908

Moenkhausia dichroura (Kner, 1858)

Moenkhausia lepidura (Kner, 1858)

Moenkhausia oligolepis (Günther, 1864)

Moenkhausia pyrophthalma Costa, 1994

Moenkhausia sp.

Moenkhausia venerei Petrolli, Azevedo-Santos \& Benine 2016

Odontostilbe sp.

Phenacogaster cf. pectinata (Cope, 1870)

Phenacogaster sp.

Psalidodon xavante Garutti \& Venere, 2009

Roeboexodon geryi Myers, 1960

Serrapinnus cf. piaba (Lütken, 1875)

Tetragonopterus chalceus Spix \& Agassiz, 1829

Tetragonopterus sp.

Thayeria boehlkei Weitzman, 1957

\section{Bryconidae}

Brycon falcatus Müller \& Troschel, 1844

Iguanodectidae

Bryconops alburnoides Kner, 1858

Bryconops giacopinii (Fernández-Yépez, 1950)

Bryconops melanurus (Bloch, 1794)

\section{Crenuchidae}

Characidium cf. zebra Eigenmann, 1909

Characidium mirim Netto-Ferreira, Birindelli \& Buckup, 2013

Characidium sp.1

Characidium sp.2

\section{SILURIFORMES}

\section{Auchenipteridae}

Trachelyopterus galeatus (Linnaeus, 1758)

\section{Pimelodidae}

Pimelodus ornatus Kner, 1858

Pseudopimelodidae

Microglanis sp.

Pseudopimelodus cf. pulcher (Boulenger, 1887)

\section{Heptapteridae}

Cetopsorhamdia sp.

Cetopsorhamdia sp.2

Imparfinis mirini Haseman, 1911

600

1

82

102

511

46
523

$\begin{array}{ccc} & 39 & 39 \\ 600 & 141 & 141 \\ & 1233 & 1833\end{array}$

91

8

224

91

27

27

2

137

234

1

4

192

1031

48

622 
...continuation

Imparfinis $\mathrm{sp.}$

Mastiglanis asopos Bockmann, 1994

Phenacorhamdia somnians (Mees, 1974)

Pimelodella sp.

Pimelodella sp.1

Pimelodella sp. 2

Pimelodella sp. 3

Rhamdia quelen (Quoy \& Gaimard, 1824)

\section{Cetopsidae}

Cetopsis coecutiens (Lichtenstein, 1819)

Cetopsis sp.

\section{Asprendinidae}

Bunocephalus sp.

Trichomycteridae

Ituglanis macunaima Datovo \& Landim, 2005

Stegophilus sp.

\section{Callichthyidae}

Aspidoras poecilus Nijssen \& Isbrücker, 1976

Callichthys callichthys (Linnaeus, 1758)

Corydoras araguaiaensis Sands, 1990

Corydoras maculifer Nijssen \& Isbrücker, 1971

\section{Loricariidae}

Ancistrus sp.1

Ancistrus sp.2

Ancistrus sp.3

Aphanotorulus emarginatus Valenciennes, 1840

Farlowella aff. oxyrryncha (Kner, 1853)

Farlowella aff. schreitmuelleri Arnold, 1936

Hisonotus sp.

Hypoptopoma gulare Cope, 1878

Hypostomus aff. cochliodon Kner, 1854

Hypostomus cf. atropinnis (Eigenmann \& Eigenmann, 1880)

Hypostomus faveolus Zawadzki, Birindelli \& Lima 2008

Hypostomus sp.1

Hypostomus sp.2

Hypostomus sp. 3

Hypostomus sp.4

Hypostomus sp.5

Loricaria sp. 1

Loricaria sp.2

Loricaria sp. 3

Otocinclus sp.

Parancistrus sp.

Parotocinclus britskii Boeseman, 1974

Parotocinclus sp.

Rineloricaria hasemani Isbrücker \& Nijssen, 1979

Sturisoma nigrirostrum Fowler, 1940
3

177

11

49

4

$$
1
$$

2

1

9

9

38

72

4

50

25

11

12

38

\section{4}

.

8

2

.


...continuation

GYMNOTIFORMES

Sternopygidae

Eigenmannia trilineata Lópes \& Castello, 1966

Sternopygus macrurus (Bloch \& Schneider, 1801)

Apteronotidae

Apteronotus albifrons (Linnaeus, 1766)

5

Apteronotus leptorhynchus (Ellis, 1912)

Rhamphichthyidae

Gymnorhamphichthys petiti Géry \& Vu, 1964

Gymnotidae

Electrophorus electricus (Linnaeus, 1766)

Gymnotus cf. carapo Linnaeus, 1758

Gymnotus sp.

\section{CYPRINODONTIFORMES}

\section{Rivulidae}

Melanorivulus zygonectes (Myers, 1927)

Plesiolebias lacerdai Costa, 1989

Poeciliidae

Pamphorichthys araguaiensis Costa, 1991

\section{SYNBRANCHIFORMES}

\section{Synbranchidae}

Synbranchus marmoratus Bloch, 1795

\section{CICHLIFORMES}

\section{Cichlidae}

Aequidens tetramerus Heckel, 1840

Apistogramma sp.

Biotodoma aff. cupido Heckel, 1840

2

Cichlasoma sp.

1

Crenicichla labrina (Spix \& Agassiz, 1831)

18

Crenicichla reticulata (Heckel, 1840)

Crenicichla sp.

Heros aff. efasciatus Heckel, 1840

Laetacara araguaiae Ottoni \& Costa, 2009

Retroculus lapidifer (Castelnau, 1855)

Retroculus sp.

1

$\begin{array}{cc}20 & 38 \\ 7 & 7\end{array}$

5

$1-1$

8

$10 \quad 10$

$6 \quad 8$

3

richness and diversity of the family in the Neotropical region (over 830 described species). Species in this family feed mainly on detritus and algae (Lujan et al. 2012). Detritus are abundant resources in tropical streams and are fundamental to ecosystem function (Bowen 1983), allowing those streams to support the high abundance and richness of detritivores fishes (Bowen 1983, Power 1983). In addition, the loricariid catfishes have an extraordinary variation in mouth morphologies, allowing them to forage detritus and periphyton in different types of habitat structures (e.g., rocks, trunks, sands, margins, and fast-water environments) presents in the headwater streams (Power 1983, Lujan et al. 2012).
Although the fish orders and families of the basin are well known, this pattern is not proper to species. We identified approximately $30 \%$ of the collected species only at the genera level. Many still need more detailed taxonomic and molecular studies (e.g., Characidium cf. zebra, Hoplias cf. malabaricus and Gymnotus cf. carapo). The significant number of species identified only at the genera level shows the need for further taxonomic studies in this basin. Considering the high fish species endemicity within these basins, there is a significant likelihood that many of these species identified only up to the genera level are new species. Previous studies on stream fish in these basins have also found similar patterns, with a great number of species not yet described 


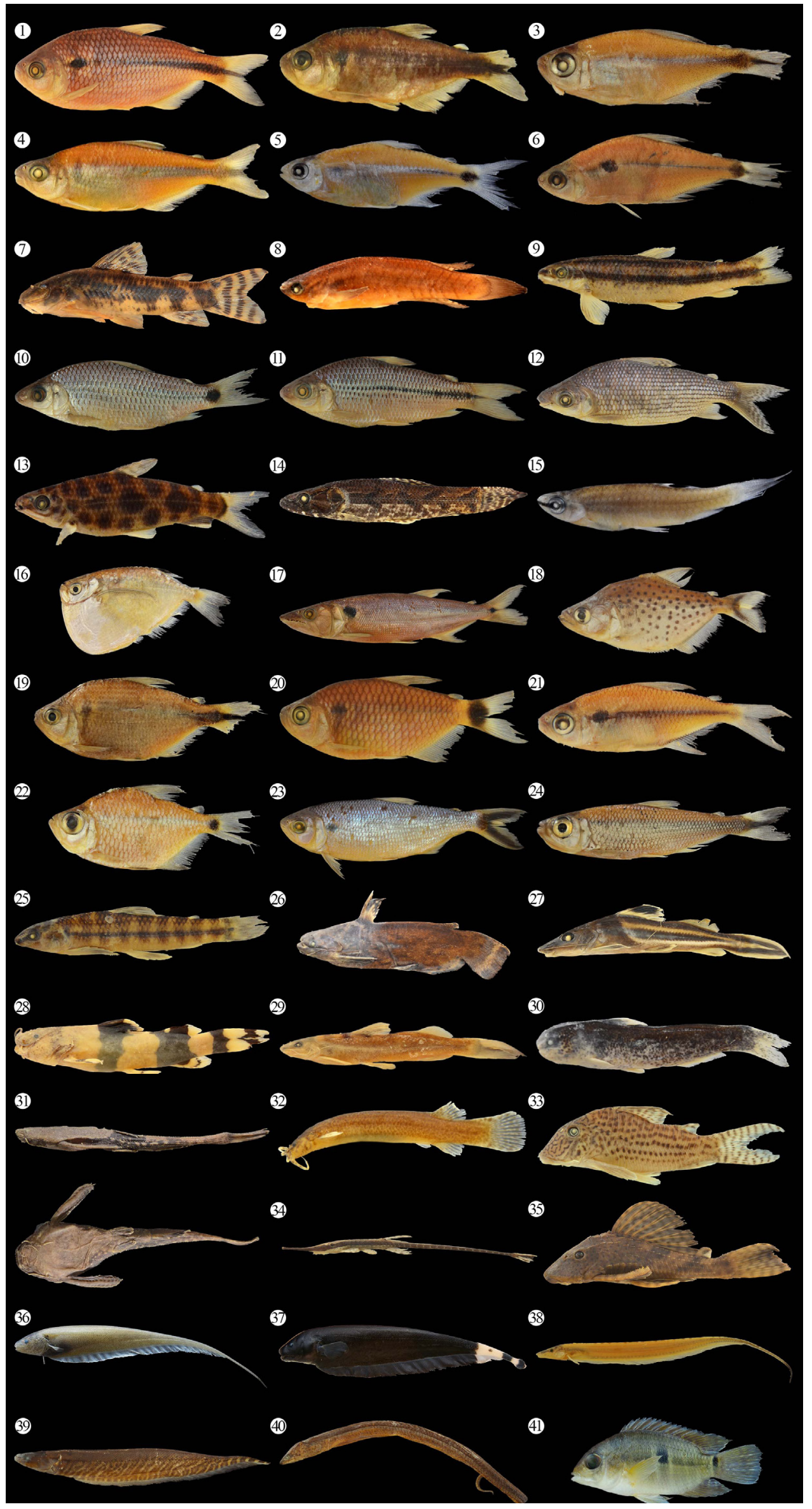


Figure 4. The eight most abundant sampled species in the Upper Araguaia and the Middle Rio das Mortes basin and representative species of each family. Some families can have more than one more species represented. The total lengths mean are presented after the names of species. 1) Astyanax cf. goyacensis, 58.9 mm; 2) Psalidodon xavante, $45.9 \mathrm{~mm}$;) Hyphessobrycon aff. tenuis, $31.4 \mathrm{~mm}$ 4) Knodus cf. breviceps, $36.6 \mathrm{~mm}$; 5) Odontostilbe sp., $30.9 \mathrm{~mm}$; 6$)$ Phenacogaster sp., $36.9 \mathrm{~mm}$; 7) Aspidoras poecilus, $31.5 \mathrm{~mm}$; 8) Melanorivulus zygonectes, $29.6 \mathrm{~mm}$. Parodontidae - 9) Parodon pongoensis, $64.6 \mathrm{~mm} ;$ Curimatidae - 10) Cyphocharax gouldingi, $71.3 \mathrm{~mm}$, 11) Steindachnerina amazonica, $105.7 \mathrm{~mm}$; Prochilondotidae - 12) Prochilodus nigricans, $262.0 \mathrm{~mm}$; Anostomidae - 13 ) Leporinus sp.1, 85.7 mm; Erythrinidae - 14) Hoplias cf. malabaricus, $106.3 \mathrm{~mm}$; Lebiasinidae - 15) Pyrrhulina australis, $37.0 \mathrm{~mm}$; Gasteropelecidae - 16) Thoracocharax cf. stellatus, $58.3 \mathrm{~mm}$; Acestrorhynchidae - 17) Acestrorhynchus falcatus, $207.5 \mathrm{~mm}$; Serrasalmidae - 18) Serrasalmus spilopleura, 63.9 mm; Characidae - 19) Moenkhausia aurantia, $57.3 \mathrm{~mm}$, 20) Moenkhausia oligolepis, $57.8 \mathrm{~mm}, 21)$ Moenkhausia venerei, $34.3 \mathrm{~mm}, 22)$ Tetragonopterus chalceus, $50.0 \mathrm{~mm}$; Bryconidae - 23) Brycon falcatus, $250.3 \mathrm{~mm}$; Iguanodectidae - 24) Bryconops giacopinii, $56.3 \mathrm{~mm}$; Crenuchidae - 25) Characidium cf. zebra, 35.2 mm; Auchenipteridae - 26) Trachelyopterus galeatus, $112.0 \mathrm{~mm}$; Pimelodidae - 27) Pimelodus ornatus, 150.5 mm; Pseudopimelodidae - 28) Pseudopimelodus cf. pulcher, $48.5 \mathrm{~mm}$; Heptapteridae - 29) Imparfinis mirini, $52.3 \mathrm{~mm}$; Cetopsidae - 30) Cetopsis coecutiens, $57.1 \mathrm{~mm}$; Asprendinidae - 31) Bunocephalus sp., $79.9 \mathrm{~mm}$; Trichomycteridae - 32) Ituglanis macunaima, $47.5 \mathrm{~mm}$; Callichthyidae - 33) Corydoras maculifer, 50.0 mm; Loricariidae - 34) Farlowella aff. oxyrryncha, $102.6 \mathrm{~mm}, 35)$ Hypostomus sp.3, $53.6 \mathrm{~mm}$; Sternopygidae - 36) Sternopygus macrurus, $197.6 \mathrm{~mm}$; Apteronotidae - 37) Apteronotus albifrons, 139.4 mm; Rhamphichthyidae - 38) Gymnorhamphichthys petiti, 143.9 mm; Gymnotidae - 39) Gymnotus cf. carapo, 150.7 mm; Synbranchidae - 40) Synbranchus marmoratus, $166.4 \mathrm{~mm}$; Cichlidae - 41) Aequidens tetramerus, $63.2 \mathrm{~mm}$.
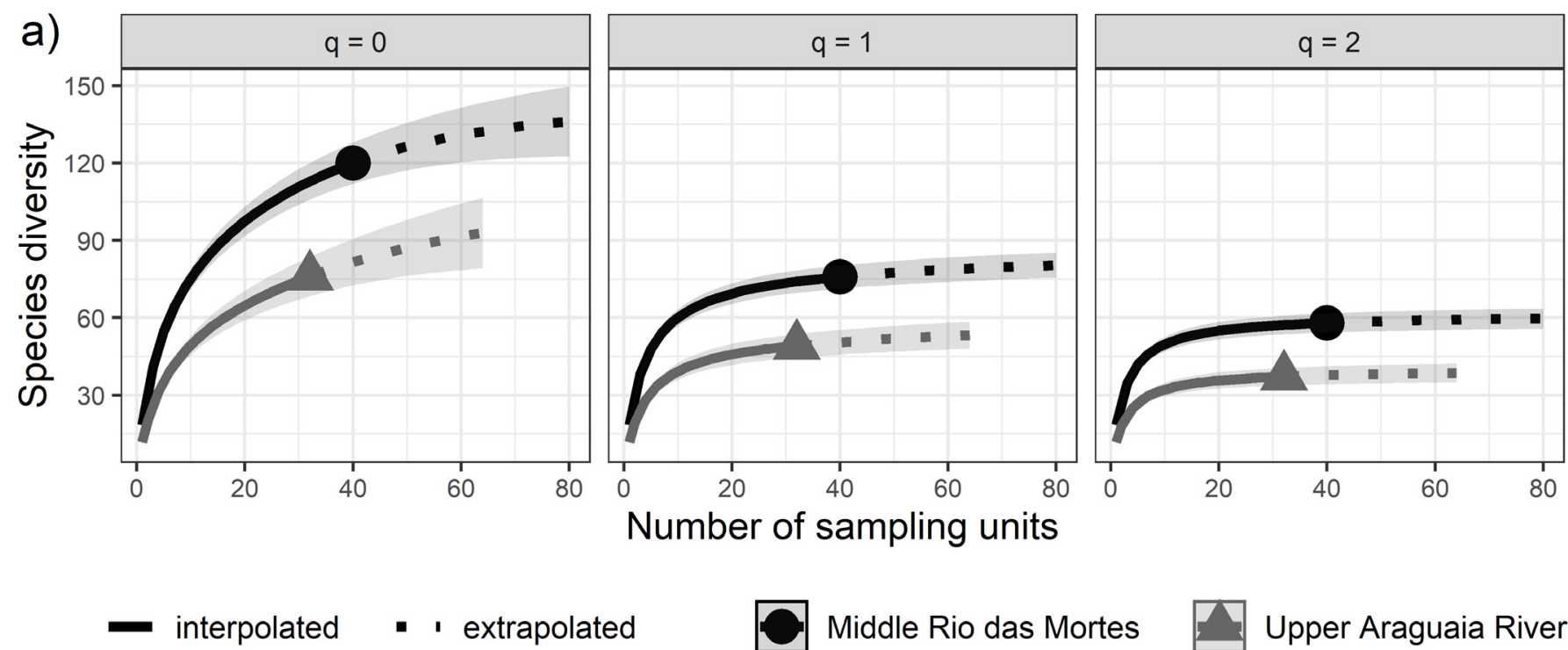

Middle Rio das Mortes

Upper Araguaia River
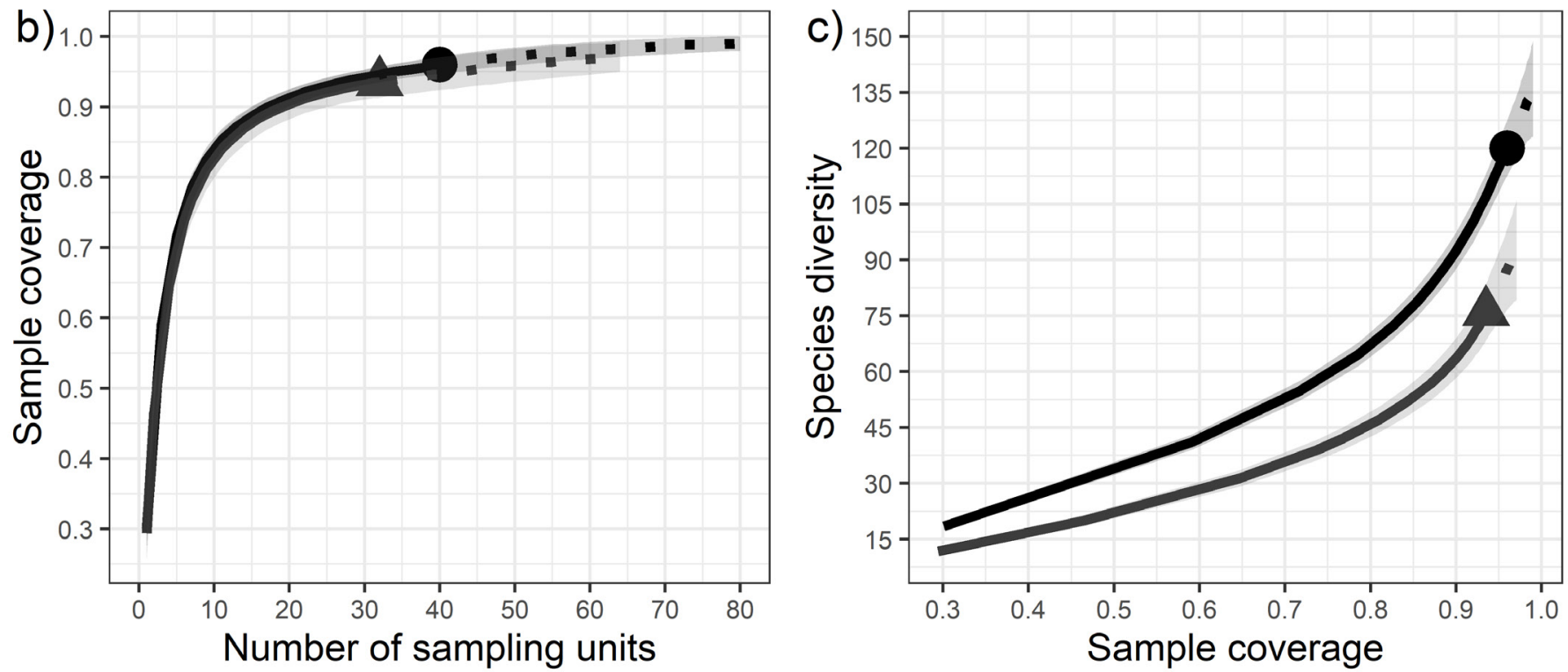

Figure 5. Sampling effort effectiveness for the Upper Araguaia River and the Middle Rio das Mortes basin, using the rarefaction method (solid line) and extrapolation (dotted line) based on Hill's numbers. (a) Incidence-based species accumulation curves, separated by order of diversity: $q=0$ (Species richness), $q=1$ (Shannon diversity) and $q=2$ (Simpson diversity). (b) Sample completeness curves based on the number of sampling sites (c) coverage-based sampling curves based on species richness. We plotted all extrapolation curves to double the sample size, and the shaded area in all figures (a-b) denotes a confidence interval at $95 \%$ obtained from a bootstrap method with 999 replications. 
(Melo et al. 2004, Matos et al. 2013, Jarduli et al. 2014, Oliveira et al. 2020). This large number of species to be described is one of the most important knowledge gaps in biodiversity (Linnean shortfalls) that needs to be addressed (Hortal et al. 2015). Even after two decades after the first studies with stream fish in the studied region, we still have a long way to know the actual diversity of stream fish in these basins. Although the number of researchers working with stream fish has increased, this advance is based less on taxonomic research than on other stream fish research (Junqueira et al. 2020, Lima 2021).

The average stream fish species richness observed in our study streams was 15 species, like the value found in other stream fish studies in this ecoregion (Melo et al. 2004, Matos et al. 2013, Barbosa et al. 2019, Oliveira et al. 2020). However, our data showed a wide variance in the number of species per stream. We sampled streams with one species and streams with up to 48 species. We believe that such variation in species richness between streams can be explained by environmental heterogeneity and hydrographic network size (Lowe-McConnell 1987, Tedesco et al. 2008, Vieira et al. 2018). The water physicochemical characteristics and habitat structures can vary spatially between nearby streams. This condition increases local environmental heterogeneity and makes fish diversity vary between streams (Benda et al. 2004). Besides, streams with more extensive drainage networks should support more diverse communities than streams with smaller drainage networks (Zbinden \& Matthews 2017).

In our study, species with wide distribution (species that occurred in more than $50 \%$ of the sites) over the sampled streams belonged to groups of fish with more diversified feeding behaviors and reproductive strategies (Winemiller 1989). For the species present in our samples, we highlight the nektonic omnivores characids that feed on materials drifting in the water column and have split spawning (Astyanax cf. goyacensis; Knodus cf. breviceps, and Moenkhausia oligolepis), the benthic invertivores with fusiform bodies (Characidium cf. zebra), and the benthic omnivores catfish (Imparfinis mirini).

The species abundance patterns we found in this study are like those observed in streams with lower environmental degradation (Ferreira \& Casatti 2006, Casatti, Ferreira \& Langeani 2009). The species rarity of our samples is relatively high, with approximately $71.5 \%$ of them having at least five individuals collected and 18 having only one sampled specimen. The rare species patterns in ecological communities vary in abundance, where few species are common, some have intermediate abundance, and many are rare (Magurran \& Henderson 2003). The number of rare species can vary from site to site because of natural variations in species abundance distributions or anthropogenic stressors (Preston 1948, Magurran \& Henderson 2011). It is important to highlight that the rarity pattern for some species found in our study differs between the basins; for example, Gymnorhamphichthys petiti was rare in Upper Araguaia, but was abundant in the Middle Rio das Mortes basin. On the other hand, Hypostomus sp. 4 was abundant in Upper Araguaia and rare in the Middle Rio das Mortes basin. Furthermore, the significant number of exclusive species in each basin shows distinct fish communities between them. Considering that the Araguaia River and Rio das Mortes belong to the same ecoregion (Abell et al. 2008), we assume that species dispersion (capacity for colonizing new habitats) and environmental filters are the main drivers responsible for the differences observed.

In this study, we did not find the fish species endangered. This finding can indicate a good ecological integrity of streams once the most sampled streams still have relatively preserved environmental conditions (Lima 2019). However, this scenario can change in the future. There is a series of threats to fish biodiversity within the basin's streams, mainly due to deforestation and the planned hydropower dams (Coe et al. 2011, Latrubesse et al. 2019, Dagosta et al. 2020, Pelicice et al. 2021). Considering the exclusive fish species of both basins, the human threats in those regions, and the few existent protected areas, we need a better look at this ecosystem's aquatic biodiversity conservation. This study contributes to filling one critical biodiversity knowledge gap (i.e., geographic distribution-Wallacean shortfalls) for fish species in streams in the Araguaia River and Rio das Mortes basins.

\section{Acknowledgments}

We are grateful UNEMAT Graduate Program in Ecology and Conservation (UNEMAT/PPGEC) and Fundação de Amparo à Pesquisa do Estado de Mato Grosso (FAPEMAT; "Projeto Veredas" 227925/2015) for financial support.. We are also grateful to colleagues at the Laboratório de Ecologia e Conservação de Ecossistemas Aquáticos (LECEA/UFMT) for help in field collect. We would like to thank all the landowners by permission to sampling collect on their properties. L.B. Lima and F. J. M. Oliveira were partially financed by the Coordenação de Aperfeiçoamento de Pessoal de Nível Superior - Brasil (CAPES) Finance Code - 001; D. P. Lima-Junior was supported by Conselho Nacional de Desenvolvimento Científico e Tecnológico (CNPq) (Grants: 448823/2014-4, 305923/2020-0).

\section{Author Contributions}

Luciano B. Lima: Substantial contribution in the concept and design of the study; contribution to data collection; contribution to data analysis and interpretation; contribution to manuscript preparation.

Fagner Junior M. Oliveira: Substantial contribution in the concept and design of the study; contribution to data collection; contribution to manuscript preparation; contribution to critical revision, adding intelectual content.

Fernando V. Borges: Substantial contribution in the concept and design of the study; contribution to data collection; contribution to manuscript preparation.

Fabiano Corrêa: Substantial contribution in the concept and design of the study; contribution to manuscript preparation; contribution to critical revision, adding intelectual content.

Dilermando P. Lima-Junior: Substantial contribution in the concept and design of the study; contribution to data collection; contribution to manuscript preparation; contribution to critical revision, adding intelectual content.

\section{Conflicts of interest}

All authors declare no competing interests.

\section{Reference}

ABELL, R. et al. 2008. Freshwater ecoregions of the world: a new map of biogeographic units for freshwater biodiversity conservation. Bioscience 58(5):403.

ALBERT, J., PETRY, P. \& REIS, R.E. 2011. Major biogeographic and phylogenetic patterns. In Historical biogeography of Neotropical freshwater fishes (J.S. Albert \& R.E. Reis, eds). University of California Press, Berkeley, p.21-56. 
ALVARES, C.A., STAPE, J.L., SENTELHAS, P.C., GONÇALVES, J.L.M. \& SPAROVEK, G. 2013. Köppen's climate classification map for Brazil. Meteorol. Zeitschrift 22(6):711-728.

AQUINO, S., LATRUBESSE, E. \& BAYER, M. 2010. Assessment of wash load transport in the Araguaia River (Aruanã gauge station), central Brazil. Lat. Am. J. Sedimentol. Basin Anal. 16(2):119-128.

BARBOSA, H. de O., BORGES, P.P., DALA-CORTE, R.B., MARTINS, P.T. de A. \& TERESA, F.B. 2019. Relative importance of local and landscape variables on fish assemblages in streams of Brazilian savanna. Fish. Manag. Ecol. 26(2):119-130.

BARLETTA, M., JAUREGUIZAR, A.J., BAIGUN, C., FONTOURA, N.F., AGOSTINHO, A.A., ALMEIDA-VAL, V.M.F., VAL, A.L., TORRES, R.A., JIMENES-SEGURA, L.F., GIARRIZZO, T., FABRE, N.N., BATISTA, V.S., LASSO, C., TAPHORN, D.C., COSTA, M.F., CHAVES, P.T., VIEIRA, J.P. \& CORRÊA, M.F.M. 2010. Fish and aquatic habitat conservation in South America: a continental overview with emphasis on Neotropical systems. J. Fish Biol. 76(9):2118-2176.

BENDA, L., POFF, N.L., MILLER, D., DUNNE, T., REEVES, G., PESS, G. \& POLLOCK, M. 2004. The network dynamics hypothesis: how channel networks structure riverine habitats. Bioscience 54(5):413-427.

BOWEN, S.H. 1983. Detritivory in neotropical fish communities. Environ. Biol. Fishes 9(2):137-144.

CASATTI, L., FERREIRA, C.P. \& CARVALHO, F.R. 2009. Grass-dominated stream sites exhibit low fish species diversity and dominance by guppies: an assessment of two tropical pasture river basins. Hydrobiologia 632(1):273-283.

CASATTI, L., FERREIRA, C.P. \& LANGEANI, F. 2009. A fish-based biotic integrity index for assessment of lowland streams in southeastern Brazil. Hydrobiologia 623(1):173-189.

CASTRO, R.M.C. 1999. Evolução da ictiofauna de riachos sul-americanos: padrões gerais e possíveis processos causais. In Ecologia de peixes de riachos (E. P. Caramaschi, R. Mazzoni, \& P. R. Peres-Neto, eds) Oecologia Brasiliensis, Rio de Janeiro, Brasil, p.139-155.

CASTRO, R.M.C. \& POLAZ, C.N.M. 2020. Small-sized fish: the largest and most threatened portion of the megadiverse neotropical freshwater fish fauna. Biota Neotrop. 20(1):313-324. https://doi.org/10.1590/1676-0611bn-2018-0683 (last access on 12/02/2021)

CETRA, M., MATTOX, G., ROMERO, P.B., ESCOBAR, S.H., GUIMARÃES, E.A., ANTONIO, R., TURIN, F., CETRA, M., MATTOX, G., ROMERO, P.B., GUIMARÃES, S.H. \& ICHTHYOFAUNA, R.A.F. 2020. Ichthyofauna from "serranias costeiras" of the Ribeira de Iguape River basin, Southeast Brazil Ictiofauna das "serranias costeiras" da bacia do rio Ribeira de Iguape, sudeste do Brasil. Biota Neotrop. 20(4):2020. https://doi.org/10.1590/16760611-bn-2020-0994 (last access on 12/02/2021)

CHAO, A., GOTELLI, N.J., HSIEH, T.C., SANDER, E.L., MA, K.H., COLWELL, R.K. \& ELLISON, A.M. 2014. Rarefaction and extrapolation with Hill numbers: a framework for sampling and estimation in species diversity studies. Ecol. Monogr. 84(1):45-67.

COE, M.T., LATRUBESSE, E.M., FERREIRA, M.E. \& AMSLER, M.L. 2011. The effects of deforestation and climate variability on the streamflow of the Araguaia River, Brazil. Biogeochemistry 105(1-3):119-131.

COLWELL, R.K., CHAO, A., GOTELLI, N.J., LIN, S.-Y., MAO, C.X., CHAZDON, R.L. \& LONGINO, J.T. 2012. Models and estimators linking individual-based and sample-based rarefaction, extrapolation and comparison of assemblages. J. Plant Ecol. 5(1):3-21.

CUMMINS, K.W. 1974. Structure and function of stream ecosystems. Bioscience 24(11):631-641.

DAGOSTA, F.C.P., PINNA, M., PERES, C.A. \& TAGLIACOLLO, V.A. 2020. Existing protected areas provide a poor safety-net for threatened Amazonian fish species. Aquat. Conserv. Mar. Freshw. Ecosyst. 1-23.

DUDGEON, D., ARTHINGTON, A.H., GESSNER, M.O., KAWABATA, Z.-I., KNOWLER, D.J., LÉVÊQUE, C., NAIMAN, R.J., PRIEUR-RICHARD, A.-H., SOTO, D., STIASSNY, M.L.J. \& SULLIVAN, C.A. 2006. Freshwater biodiversity: importance, threats, status and conservation challenges. Biol. Rev. 81(2):163-182.
FERREIRA, C.D.P. \& CASATTI, L. 2006. Influência da estrutura do hábitat sobre a ictiofauna de um riacho em uma micro-bacia de pastagem, São Paulo, Brasil. Rev. Bras. Zool. 23(3):642-651.

FRICKE, R., ESCHMEYER, W.N. \& VAN DER LAAN, R. 2020. Catalog of fishes: genera, species, references. http://researcharchive.calacademy.org/ research/ichthyology/catalog/fishcatmain.asp. (last access on 12/02/2021).

HILL, M.O. 1973. Diversity and evenness: a unifying notation and its consequences. Ecology 54(2):427-432.

HORTAL, J., DE BELLO, F., DINIZ-FILHO, J.A.F., LEWINSOHN, T.M., LOBO, J.M. \& LADLE, R.J. 2015. Seven shortfalls that beset large-scale knowledge of biodiversity. Annu. Rev. Ecol. Evol. Syst. 46(1):523-549.

HSIEH, T.C., MA, K.H. \& CHAO, A. 2016. iNEXT: an R package for rarefaction and extrapolation of species diversity (Hill numbers) G. McInerny, ed. Methods Ecol. Evol. 7(12):1451-1456.

ICMBio, 2020. Instituto Chico Mendes de Conservação da Biodiversidade. Cerrado. https://www.icmbio.gov.br/portal/unidadesdeconservacao/biomasbrasileiros/cerrado (last access on 28/12/2020)

INMET, 2020. Instituto Nacional de Meteorologia. http://www.inmet.gov.br/ portal/ (last access on 17/08/2020)

JARDULI, L.R., CLARO-GARCÍA, A. \& SHIBATTA, O.A. 2014. Ichthyofauna of the rio Araguaia basin, states of Mato Grosso and Goiás, Brazil. Check List 10(3):483-515.

JUNQUEIRA, N.T., MAGNAGO, L.F. \& POMPEU, P.S. 2020. Assessing fish sampling effort in studies of Brazilian streams. Scientometrics 123(2):841-860.

LATRUBESSE, E.M., AMSLER, M.L., MORAIS, R.P. \& AQUINO, S. 2009. The geomorphologic response of a large pristine alluvial river to tremendous deforestation in the South American tropics: The case of the Araguaia River. Geomorphology 113(3-4):239-252.

LATRUBESSE, E.M., ARIMA, E., FERREIRA, M.E., NOGUEIRA, S.H., WITTMANN, F., DIAS, M.S., DAGOSTA, F.C.P. \& BAYER, M. 2019. Fostering water resource governance and conservation in the Brazilian Cerrado biome. Conserv. Sci. Pract. 1(9):1-8.

LATRUBESSE, E.M. \& STEVAUX, J.C. 2002. Geomorphology and environmental aspects of the Araguaia fluvial basin, Brazil. Zeitschrift fur Geomorphol. Suppl. 129(December 2015):109-127.

LEAL, C.G., JUNQUEIRA, N.T., CASTRO, M.A., CARVALHO, D.R., FAGUNDES, D.C., SOUZA, M.A., HUGHES, R.M. \& POMPEU, P.S. 2014. Ichthyofaunal structure of Cerrado streams in Minas Gerais. In Ecological conditions in hydropower basins (M. Callisto, R. M. Hughes, R. M. Lopes, \& M. A. Castro, eds) Companhia Energética de Minas Gerais, Belo Horizonte, p.101-126.

LIMA, J.D. 2009. Conectividade e análise da estrutura taxonômica e trófica da ictiofauna em lagos do rio das Mortes, Mato Grosso-Brasil. Tese de doutorado, Universidade Federal de São Carlos, São Carlos.

LIMA, L.B. 2019. Da cienciometria ao campo: fatores que estruturam as comunidades de peixes em riachos. Tese de doutorado, Universidade do Estado do Mato Grosso, Nova Xavantina.

LIMA, L.B., DE MARCO JÚNIOR, P. \& LIMA-JUNIOR, D.P. 2021. Trends and gaps in studies of stream-dwelling fish in Brazil. Hydrobiologia. https:// doi.org/10.1007/s10750-021-04616-8

LOWE-MCCONNELL, R. 1999. Estudos Ecológicos de Comunidades de Peixes Tropicais. EDUSP, São Paulo.

LUJAN, N.K., WINEMILLER, K.O. \& ARMBRUSTER, J.W. 2012. Trophic diversity in the evolution and community assembly of loricariid catfishes. BMC Evol. Biol. 12(1):124

MAGURRAN, A.E. \& HENDERSON, P.A. 2003. Explaining the excess of rare species in natural species abundance distributions. Nature 422(6933):714 716.

MAGURRAN, A.E. \& HENDERSON, P.A. 2011. Commonness and rarity. In Biological Diversity: Frontiers in Measurement and Assessment (A. E. Magurran \& B. J. McGill, eds) Publisher: Oxford University Press, New Jersey, p.97-104. 
MATOS, P.R., CARMO, C.M. \& MELO, C.E. 2013. Relação entre variáveis ambientais e a estrutura da comunidade de peixes em córregos das bacias do Rio das Mortes e do rio Xingu - MT, Brasil. Biotemas 26(3):139-151.

MELO, C.E., MACHADO, F.D.A. \& PINTO-SILVA, V. 2004. Feeding habits of fish from a stream in the savanna of Central Brazil, Araguaia Basin. Neotrop. Ichthyol. 2(1):37-44.

MENDONÇA, F.P., MAGNUSSON, W.E. \& ZUANON, J. 2005. Relationships between habitat characteristics and fish assemblages in small streams of Central Amazonia. Copeia 2005(4):751-764.

MORAIS, R.P., AQUINO, S. \& LATRUBESSE, E.M. 2008. Controles hidrogeomorfológicos nas unidades vegetacionais da planície aluvial do rio Araguaia, Brasil. Acta Sci. Biol. Sci. 30(4):411-421.

MYERS, N., MITTERMEIER, R.A., MITTERMEIER, C.G., FONSECA, G.A \& KENT, J. 2000. Biodiversity hotspots for conservation priorities. Nature 403(6772):853-8.

NOGUEIRA, C., BUCKUP, P.A., MENEZES, N.A., OYAKAWA, O.T., KASECKER, T.P., RAMOS NETO, M.B. \& DA SILVA, J.M.C. 2010. Restricted-range fishes and the conservation of Brazilian freshwaters. PLoS One 5(6):e11390.

NOVOTNÝ, V. \& BASSET, Y. 2000. Rare species in communities of tropical insect herbivores: pondering the mystery of singletons. Oikos 89(3):564-572.

OKSANEN, J., BLANCHET, F.G., KINDT, R., PIERRE, L., MINCHIN, P.R., O'HARA, R.B., SIMPSON, G.L., SOLYMOS, P., STEVENS, M.H.H. \& WAGNER, H. 2018. Vegan: community ecology package. R Package. version 2.5-2.

OLIVEIRA, F.J.M., LIMA-JUNIOR, D.P. \& BINI, L.M. 2020. Current environmental conditions are weak predictors of fish community structure compared to community structure of the previous year. Aquat. Ecol. 54(3):729-740.

PEASE, A.A., GONZÁLEZ-DÍAZ, A.A., RODILES-HERNÁNDEZ, R. \& WINEMILLER, K.O. 2012. Functional diversity and trait-environment relationships of stream fish assemblages in a large tropical catchment. Freshw. Biol. 57(5):1060-1075.

PELICICE, F.M. et al. 2021. Large-scale degradation of the Tocantins-Araguaia River basin. Environ. Manage. https://doi.org/10.1007/s00267-021-01513-7.

POWER, M.E. 1983. Grazing responses of tropical freshwater fishes to different scales of variation in their food. Environ. Biol. Fishes 9(2):103-115.

PRESTON, F.W. 1948. The commonness, and rarity, of species. Ecology 29(3):254-283.

R CORE TEAM. 2019. R: A Language and environment for statistical computing R Foundation for Statistical Computing, Vienna, Austria. https://www.rproject.org/.
REID, A.J., CARLSON, A.K., CREED, I.F., ELIASON, E.J., GELL, P.A., JOHNSON, P.T.J., KIDD, K.A., MACCORMACK, T.J., OLDEN, J.D., ORMEROD, S.J., SMOL, J.P., TAYLOR, W.W., TOCKNER, K., VERMAIRE, J.C., DUDGEON, D. \& COOKE, S.J. 2019. Emerging threats and persistent conservation challenges for freshwater biodiversity. Biol. Rev. 94(3):849-873.

REIS, R.E., ALBERT, J.S., DI DARIO, F., MINCARONE, M.M., PETRY, P. \& ROCHA, L.A. 2016. Fish biodiversity and conservation in South America J. Fish Biol. 89(1):12-47.

STRAHLER, A.N. 1957. Quantitative analysis of watershed geomorphology. Eos, Trans. Am. Geophys. Union 38(6):913-920.

TEDESCO, P.A., HUGUENY, B., OBERDORFF, T., DÜRR, H.H., MÉRIGOUX, S. \& DE MÉRONA, B. 2008. River hydrological seasonality influences life history strategies of tropical riverine fishes. Oecologia 156(3):691-702.

TERESA, F.B. \& CASATTI, L. 2012. Influence of forest cover and mesohabitat types on functional and taxonomic diversity of fish communities in Neotropical lowland streams. Ecol. Freshw. Fish 21(3):433-442.

UEIDA, V.S. \& CASTRO, R.M.C. 1999. Coleta e fixação de peixes de riachos. In Ecologia de peixes de riachos (E. P. Caramaschi, R. Mazzoni, \& P. R. Peres-Neto, eds) Oecologia Brasiliensis, Rio de Janeiro, Brasil, p.1-22.

VENERE, P.C. \& GARUTTI, V. 2011. Peixes do Cerrado: Parque Estadual da Serra Azul, rio Araguaia, MT. RiMa, São Carlos.

VIEIRA, T.B. et al. 2018. A multiple hypothesis approach to explain species richness patterns in Neotropical stream-dweller fish communities L. U. Hepp, ed. PLoS One 13(9):e0204114.

WICKHAM, H. 2009. ggplot2: elegant graphics for data analysis. Springer New York, New York, NY.

WINEMILLER, K.O. 1989. Patterns of variation in life history among South American fishes in seasonal environments. Oecologia 81(2):225-241.

WINEMILLER, K.O., AGOSTINHO, A.A. \& CARAMASCHI, É.P. 2008. Fish ecology in tropical streams. In Tropical Stream Ecology (D. Dudgeon, ed.) Elsevier, San Diego, p.107-146.

ZBINDEN, Z.D. \& MATTHEWS, W.J. 2017. Beta diversity of stream fish assemblages: partitioning variation between spatial and environmental factors. Freshw. Biol. 62(8):1460-1471.

ZENI, J.O., PÉREZ-MAYORGA, M.A., ROA-FUENTES, C.A., BREJÃO, G.L. \& CASATTI, L. 2019. How deforestation drives stream habitat changes and the functional structure of fish assemblages in different tropical regions. Aquat. Conserv. Mar. Freshw. Ecosyst. aqc.3128.

Received: $18 / 02 / 2021$

Accepted: 05/08/2021

Published online: $30 / 08 / 2021$ 